\title{
Detection of Optic Disc in Retinal Images by Means of a Geometrical Model of Vessel Structure
}

\author{
M. Foracchia, E. Grisan, and A. Ruggeri*, Senior Member, IEEE
}

\begin{abstract}
We present here a new method to identify the position of the optic disc (OD) in retinal fundus images. The method is based on the preliminary detection of the main retinal vessels. All retinal vessels originate from the $\mathrm{OD}$ and their path follows a similar directional pattern (parabolic course) in all images. To describe the general direction of retinal vessels at any given position in the image, a geometrical parametric model was proposed, where two of the model parameters are the coordinates of the OD center. Using as experimental data samples of vessel centerline points and corresponding vessel directions, provided by any vessel identification procedure, model parameters were identified by means of a simulated annealing optimization technique. These estimated values provide the coordinates of the center of OD. A Matlab ${ }^{\circledR}$ prototype implementing this method was developed. An evaluation of the proposed procedure was performed using the set of 81 images from the STARE project, containing images from both normal and pathological subjects. The OD position was correctly identified in 79 out of 81 images $(98 \%)$, even in rather difficult pathological situations.
\end{abstract}

Index Terms-Geometrical model, optic disc, simulated annealing, retinal images, vessel tracking.

\section{INTRODUCTION}

$\mathbf{T}$ HE OPTIC DISC (OD), which in fundus images usually appears as a round region brighter than the surrounding, is the image of the optic nerve (Fig. 1). From it, the central retinal artery and vein emerge, to cover, with further branching, most of the retinal region. Locating the OD position in fundus images is quite important for many reasons. Many important retinal pathologies may affect the optic nerve. Since the OD may be easily confounded with large exudative lesions by image analysis techniques, its detection is also important to exclude it from the set of possible lesions. Moreover, OD detection is fundamental for establishing a frame of reference within the retinal image and is, thus, important for any image analysis application. The detection of OD position is also a prerequisite for the computation of some important diagnostic indexes for hypertensive/sclerotic retinopathy based on vasculature, such as central retinal artery equivalent (CRAE) and central retinal vein equivalent (CRVE) [1].

Manuscript received December 1, 2003; revised April 1, 2004. This work was supported in part by a grant from Nidek Technologies, Italy. The Associate Editor responsible for coordinating the review of this paper and recommending its publication was A. H. Hielscher. Asterisk indicates corresponding author.

M. Foracchia was with the Department of Information Engineering, University of Padova, 35131 Padova, Italy. He is now with M2 Scientific Computing, Reggio Emilia, 42038, Italy.

E. Grisan is with the Department of Information Engineering, University of Padova, 35131 Padova, Italy.

*A. Ruggeri is with the Department of Information Engineering, University of Padova, Via Gradenigo 6/a, 35131 Padova, Italy (e-mail: alfredo.ruggeri@unipd.it).

Digital Object Identifier 10.1109/TMI.2004.829331

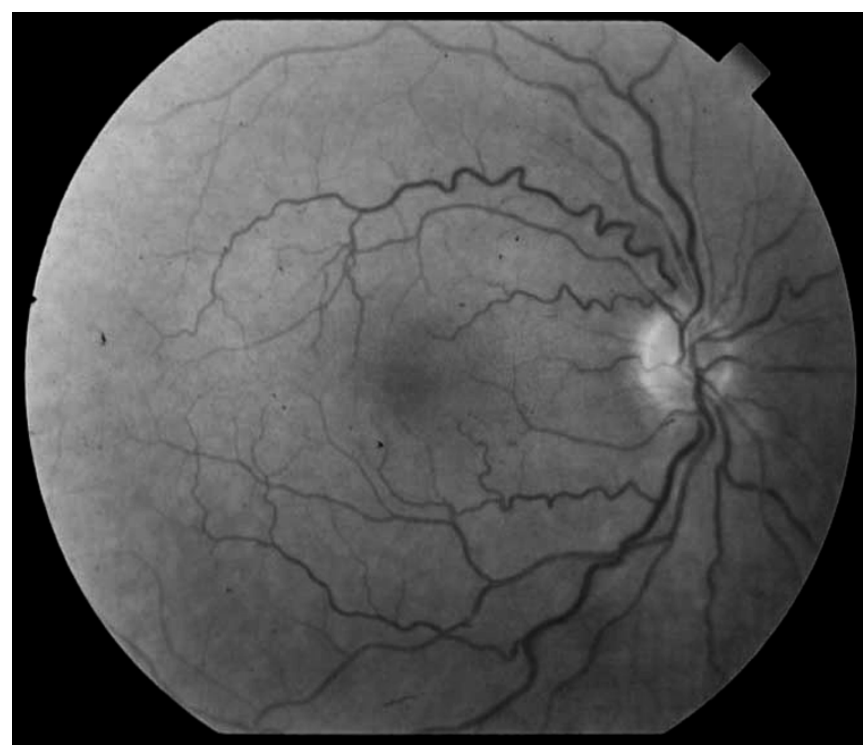

Fig. 1. Retinal fundus image with vessels and OD (bright round shape on the right-hand side).

Many techniques have been proposed to detect the OD, mainly based on its specific round shape and relatively high brightness, as compared to the rest of the fundus image (see, e.g., [2]-[7]). These techniques, however, often fail on pathological images, where other regions of fundus may be characterized by round shape and/or elevated brightness, e.g., large exudative lesions.

Other techniques have been recently proposed, which try to exploit the information provided by the vessel structure, i.e., the fact that all retinal vessels originate from the OD. In Koozekanani et al. [8], an OD tracking technique was developed for OCT (Optical Coherent Tomography) images, using a tiered scheme based on the Hough transform, eigenimage analysis and geometrical analysis based on a vasculature model. In Hoover and Goldbaum [9], an original vessel segments fuzzy convergence algorithm was proposed to identify the position of the optic nerve image as the focal point of the blood vessel network. Their method achieved $89 \%$ of correct identifications on an image data set developed within the STARE project and containing many pathological images [10]. It is the same data set we have used also in the work presented here.

Our proposed method is based on a model of the geometrical directional pattern of the retinal vascular system, which implicitly embeds the information on the OD position as the point of convergence of all vessels. However, the resulting method is not just based on the detection of the area of convergence of vessels (as in [9]), but rather on the fitting of a model with respect to the entire vascular structure. 


\section{METHODS}

\section{A. A Geometrical Model of Retinal Vessels Direction}

Defining a directional model for retinal vessels requires the definition on the whole image of a function

$$
\theta^{\bmod }(x, y ; \mathbf{p}) \quad-\frac{\pi}{2} \leq \theta^{\bmod } \leq \frac{\pi}{2},
$$

which represents the preferential direction in any retinal image of a vessel present at point $(x, y)$. Vector $\mathbf{p}$ is the set of parameters defining the model and its positioning and, thus, it will include the OD coordinates.

By visual inspection of retinal fundus images (see a representative example in Fig. 1), it appears that a common vascular pattern is present among images: the main vessels originate from the OD and follow a specific course that can be geometrically modeled as two parabolas, with a common vertex inside the OD. The definition of the directional model can, therefore, be based on this assumption.

If we assume a Cartesian coordinate system, these parabolas can be described as the geometrical locus $\Gamma$

$$
\Gamma=\left\{(x, y): a y^{2}=|x|\right\}
$$

where $a$ is the parameter governing the aperture of the parabolas (for sake of simplicity, let us assume for the time being that the origin of the coordinate system is the vertex of the parabolas). Fig. 2 shows an example of one such locus overlapped to the retinal image.

For a generic point $(x, y)$ belonging to locus $\Gamma$, i.e., on the parabola, the directional model is expressed by the implicit equation

$$
\tan \left(\theta^{\bmod }(x, y ; \mathbf{p})\right)=\operatorname{sgn}(x) \operatorname{sgn}(y) \frac{1}{2 a \sqrt{\frac{|x|}{a}}} \quad \begin{gathered}
(x, y) \in \Gamma \\
x \neq 0
\end{gathered}
$$

where function $\operatorname{sgn}()$ returns the sign of its argument and vector p contains parameter $a$. The above expression states that on the parabolas the preferential vessel direction is tangent to the parabolas themselves.

In order to completely define the model, it is necessary to express the preferential direction also outside of the parabolic geometrical locus. $\Gamma$ implicitly divides every quadrant in two areas: the internal area (with respect to the convexity of the parabola) and the external area. Anatomical knowledge indicates that vessels bifurcate when moving away from the OD, and branch vessels tend to diverge from the main vessel. In particular, vessels inside the parabolas quickly bend toward the macula in the temporal region (left-hand side in Fig. 2), whereas in the nasal region this inward deflection happens at a much slower rate (right-hand side in Fig. 2).

The tangent equation (2) was, thus, extended to accommodate points outside $\Gamma$ by adding a correction term $d$

$$
\begin{aligned}
d(x, y ; \mathbf{p}) & =\frac{y-\operatorname{sgn}(y) \sqrt{\frac{|x|}{a}}}{c(x)} \\
c(x ; \mathbf{p}) & =\frac{c_{1}}{1+e^{-x}}+\frac{c_{2}}{1+e^{x}} \quad c_{1}>0 ; \quad c_{2}<0 .
\end{aligned}
$$

The numerator of (3) is zero for a point belonging to $\Gamma$, whereas for a point outside $\Gamma$ its absolute value increases in a way proportional to the vertical distance between the point and $\Gamma$. This

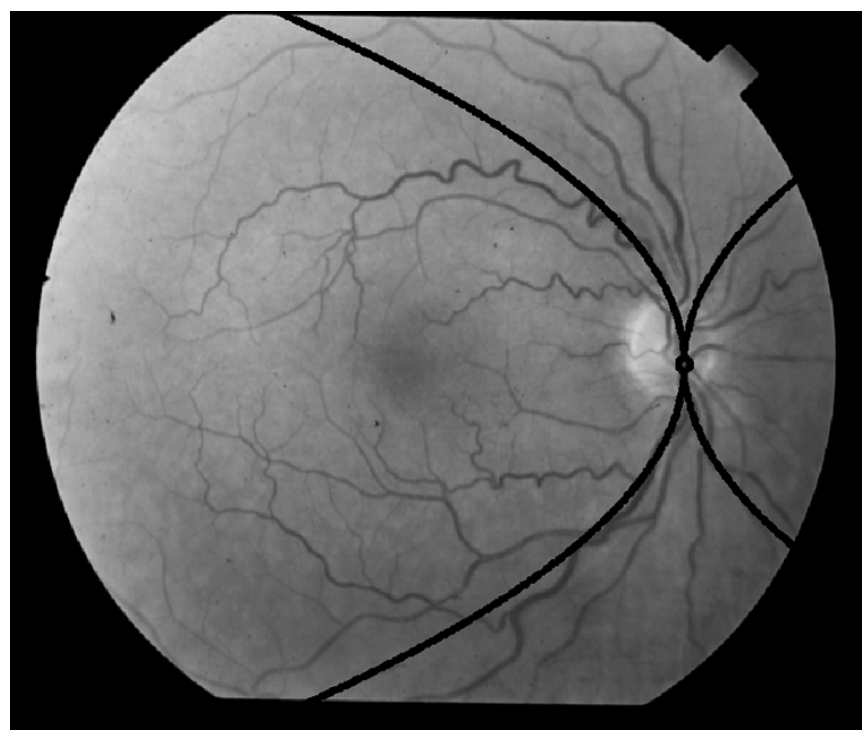

Fig. 2. Parabolic model of main vessels course.

increment in tangent magnitude is modulated by expression (4), which expresses the rate of divergence of the direction at any given $x$ coordinate. For increasing $|x|$, this rate tends toward the value of parameter $c_{1}\left(c_{2}\right)$ for positive (negative) values of $x$. Values of $c_{1}$ and $c_{2}$ represent, therefore, the limit rates of convergence toward $\pi / 2$ (vertical direction) of the vessel directions for positive and negative $\mathrm{x}$ values. These two rates are in principle different, to take care of the different degree of curvature of vessels in the nasal and temporal side of retina: the lower the absolute value of this constant, the higher the curvature of vessels as they move away from the OD.

Given a generic origin for the Cartesian coordinates system in use (e.g., upper-left corner in the image), in order for the parabolas to be centered at the coordinates of the OD center $\left(x_{\mathrm{OD}}, y_{\mathrm{OD}}\right)$, as shown in Fig. 2, a translation transformation had to be applied to the model

$$
\begin{aligned}
& x^{*}=x-x_{\mathrm{OD}} \\
& y^{*}=y-y_{\mathrm{OD}} .
\end{aligned}
$$

The complete model for vessel direction $\theta^{\bmod }$ at any point $(x, y)$ in the image is given by the following equation:

$$
\begin{aligned}
\theta^{\bmod }(x, y ; \mathbf{p}) & =\arctan \left\{\frac{\operatorname{sgn}\left(x-x_{\mathrm{OD}}\right) \operatorname{sgn}\left(y-y_{\mathrm{OD}}\right)}{2 a \sqrt{\frac{\left|x-x_{\mathrm{OD}}\right|}{a}}}\right. \\
& \left.+\frac{\left(y-y_{\mathrm{OD}}\right)-\operatorname{sgn}\left(y-y_{\mathrm{OD}}\right) \sqrt{\frac{\left|x-x_{\mathrm{OD}}\right|}{a}}}{\frac{c_{1}}{\left.1+e^{-(x-x} \mathrm{OD}\right)}+\frac{c_{2}}{1+e^{\left(x-x_{\mathrm{OD}}\right)}}}\right\} .
\end{aligned}
$$

Fig. 3 shows an example of one such model overlapped to the retinal image. For sake of clarity, directions $\theta^{\bmod }$ are shown only for some points of the image and optimal values for model parameters are used for this simulation (see below).

\section{B. Model Parameter Identification}

By using suitable model parameter identification techniques, the optimal value for $\mathbf{p}$ and, thus, for $\left(x_{\mathrm{OD}}, y_{\mathrm{OD}}\right)$, can be identified for any image, given a set of data. The data are the vessel di- 


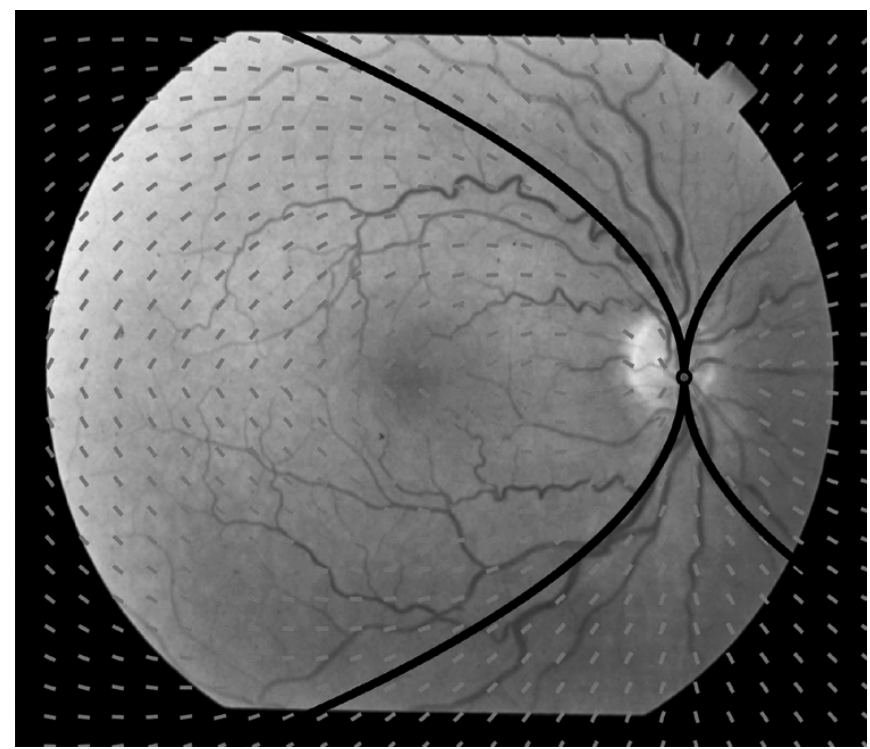

Fig. 3. Complete model of vessels direction. For sake of clarity, directions $\theta^{\mathrm{mod}}$ (gray segments) are shown only on an arbitrary grid of points.

rections $\theta_{i}$ measured at points $\left(x_{i}, y_{i}\right), i=1, \ldots, N$, belonging to the vascular structure.

Many algorithms have been developed for extracting the vascular structure from fundus images (see, e.g., [11]-[13]). Most of them provide, in addition to parameters such as vessel center-point position and caliber, also vessel direction at the center-point (this latter parameter can however be easily recovered from the identified vessel, e.g., by means of simple Principal Component Analysis on a set of vessel center-points). The detected vascular tree can, therefore, be represented by a set of quadruplets $\left(x_{i}, y_{i}, c_{i}, \theta_{i}\right)$, whose elements represent respectively the coordinates of vessel center-point $\left(x_{i}, y_{i}\right)$, the vessel caliber $c_{i}$ and vessel direction $\theta_{i}$ at that point.

Our choice for the identification of model parameters has been the minimization of the weighted residual sum of squares (RSS)

$$
\mathrm{RSS}=\sum_{i} w_{i}\left[\theta\left(x_{i}, y_{i}\right)-\theta^{\bmod }\left(x_{i}, y_{i} ; \mathbf{p}\right)\right]^{2}
$$

Minimization is performed with respect to model parameters $\mathbf{p}$ and operator "-" indicates a modulus- $\pi$ difference between directions.

Quantities $w_{i}$ are weights, used to modulate the importance of each term in the summation. Different options have been investigated to describe these weights and the best results were obtained with $w_{i}$ proportional to vessel caliber $c_{i}$. Optimized values of parameters $\left(x_{\mathrm{OD}}, y_{\mathrm{OD}}\right)$ represent the best positioning of the OD according to the model fit on the available data $\left(x_{i}, y_{i}, c_{i}, \theta_{i}\right)$.

Minimization of RSS with classical gradient-based techniques is rather critical, since this function exhibits many local minima. Fig. 4 represents, e.g., a plot of RSS as a function of parameters $x_{\mathrm{OD}}$ and $y_{\mathrm{OD}}$ only. The absolute minimum is correctly found when $\left(x_{\mathrm{OD}}, y_{\mathrm{OD}}\right)$ are inside the OD, but a gradient-based algorithm would be easily trapped in one of the many local minima. To overcome this problem, a simulated annealing (SA) optimization algorithm has been adopted. SA is a global stochastic optimization algorithm that theoretically guarantees the convergence toward global minimum [14].

The working parameters of this procedure (e.g., number of data points, initial model parameters value, initial temperature, termination criterion, etc.) have been empirically tuned using a representative subset of 20 images. The resulting set of values was then used for model parameter estimation in all 81 images of the entire data set.

In order to overcome the stochastic nature of SA algorithm, several optimization runs were performed, starting the procedure from different points in the parameter space, and the final RSS values were compared to select the smallest one. A number of six runs for each image proved to be sufficient in our test set.

\section{RESULTS}

A Matlab ${ }^{\circledR}$ prototype implementing the described method was realized. An evaluation of the proposed procedure was performed using the 81 fundus images of the STARE project data set $\left(35^{\circ}\right.$ field of view and $700 \times 605$ pixels) [10]. Thirty-one images were from normal subjects, whereas the other 50 contained pathological lesions of various types and severity.

In order to assess the robustness of the proposed procedure to detect OD position with respect to different vessel detection algorithms, we have used the measured vessel directions provided by two sets of vessel data. The first one (Track-1) was obtained by applying a binary segmentation procedure developed by Hoover et al. [12] and used to provide input data also to their own system for OD detection [9]. For each image, 10 different sets of vessel structure data were provided, to produce vessel segmentations at different scales, from 0 to 9 . At variance with [9], where 6 segmentations for each image were used to detect vessel convergence and, thus, OD detection, we used only one segmentation (scale value of 4). The second set of vessel data (Track-2) was obtained by applying our own procedure, based on a sparse tracking algorithm [13]. As proposed also in [9], the OD position was considered correctly detected if the estimated coordinates were inside the contour of the OD, i.e., within 60 pixels of its center, as manually identified for ground truth.

The method, using either set of vessel data, was able to correctly position the OD in 79 out of 81 images. Identification results are summarized in Table I for both vessel detection procedures. Examples of highly pathological images in which the OD was successfully identified are shown in Fig. 5.

SA runs took on average $2 \mathrm{~min}$ for each image on a mid-size PC (2-MHz Intel ${ }^{\circledR}$ Pentium ${ }^{\circledR}$ IV CPU and 512 Mb RAM). This value is expected to be at least ten-fold reduced when a more efficient $\mathrm{C}++$ implementation of the whole procedure will be developed.

\section{DISCUSSION}

We have developed a new algorithm for the OD detection in retinal images. It is based on a geometrical model of the direction of main retinal vessels (two parabolas with a common vertex) and its robustness lies in the a priori knowledge provided by this model. The model parameters are identified from a set of data, representing the directions of retinal vessels on a set of image points, through the minimization of a least-squares cost function. 


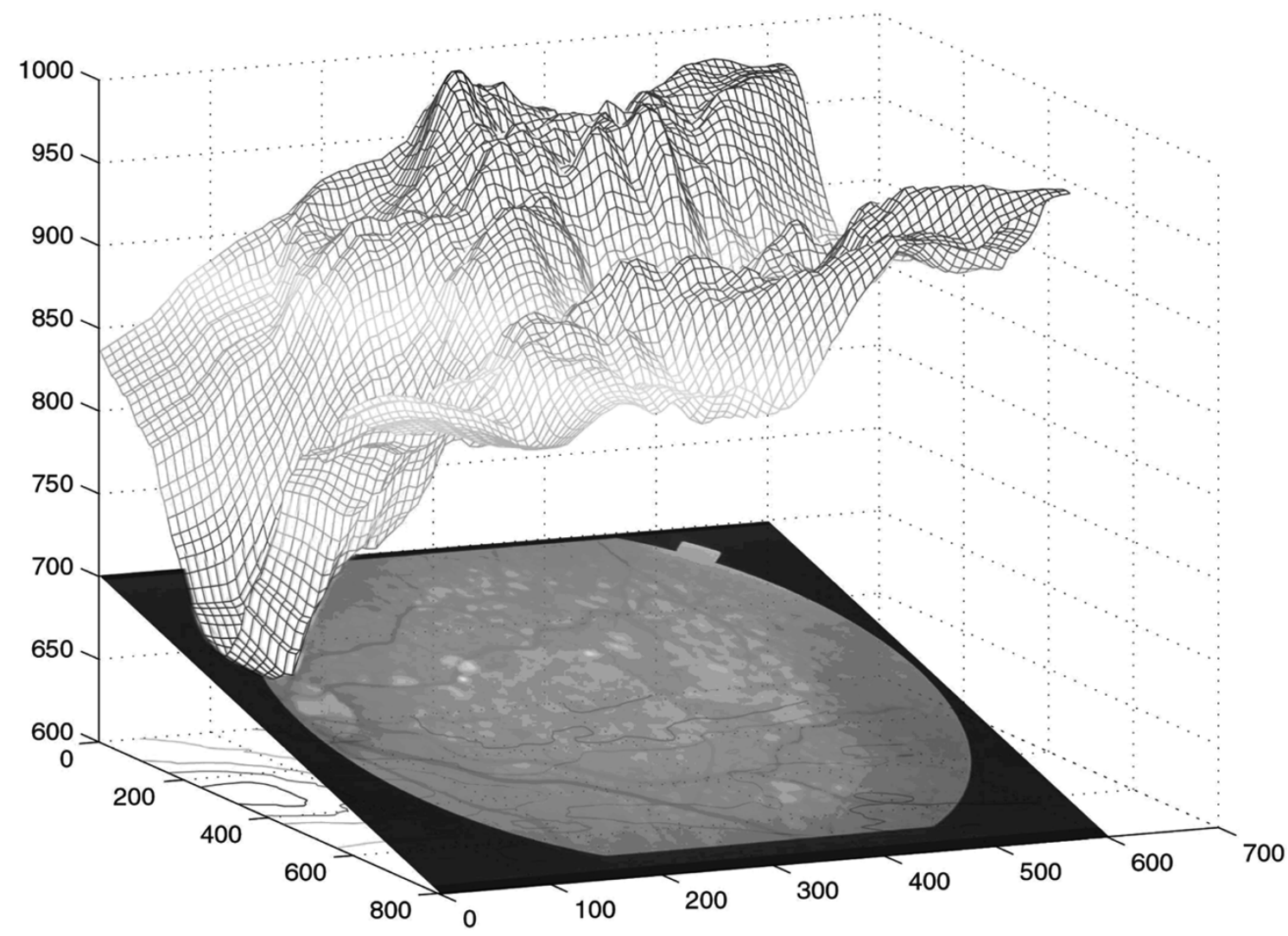

Fig. 4. Plot of RSS values (vertical axis, in arbitrary units) as a function of model parameters $x_{\mathrm{OD}}$ and $y_{\mathrm{OD}}$ (image plane, in pixels).

TABLE I

PERFORMANCES OF THE ALGORITHM, EXPRESSED AS NUMBER OF IMAGES IN WHICH THE OD WAS CORRECTLY IDENTIFIED OR NOT. IN “TRACK-1," VESSEL IDENTIFICATION DATA FROM HOOVER et al. [12] WERE USED, WHEREAS IN "Track-2" OUR OWn Tracking Algorithm [13] Was EMPLOYED

\begin{tabular}{l|l|l}
\hline & Track-1 & Track-2 \\
\hline OD identified & 79 & 79 \\
\hline OD not identified & 2 & 2 \\
\hline Total & 81 & 81 \\
\hline
\end{tabular}

The model assumes the symmetry of the vascular network around the parabolas axes, lying horizontally and, thus, images with vessels having this directional symmetry are required to obtain a reliable estimate of the OD location. This is reasonable for the fundus images acquired in clinical settings, with fields of view including OD (such as standard ETDRS fields $1 \mathrm{M}$ and 2M [15] and proposed modified fields NM1 and NM3 [16]). The vessels identified in the image should also have sufficient curvature to provide good sensitivity of the model parameters to the data.

A preliminary investigation was performed to assess the adequacy of the proposed model by analyzing the behavior of the cost function RSS when model parameter values are varied. These values were moved one by one on their interval of variation and the existence of an absolute minimum for RSS was confirmed. As an example, the values assumed by RSS for parameters $x_{\mathrm{OD}}$ and $y_{\mathrm{OD}}$ varying over a grid of coordinates are shown in Fig. 4; albeit many local minima are present, a global minimum exists and it is reached just for $x_{\mathrm{OD}}$ and $y_{\mathrm{OD}}$ at the center of OD. Moreover, while two different values for parameter $c$ were found to be necessary ( $c_{1}$ and $c_{2}$ ), only one value for parameter $a$ turned out to be adequate for obtaining satisfactory performances of the algorithm.

The issue of the number of data points $N$ to be used in each image for parameter estimation has been also examined. The number of points originally provided by the vessel identification procedures ranged from 4300 to 7800 , depending on the quality of the images and the parameters used in the procedures (e.g., scale value in "Track-1"). To reduce the computational burden of the algorithm, an analysis was performed to identify an average minimum number of data points. In the subset of $20 \mathrm{im}-$ ages, we investigated how the reduction in $N$ affected the position of RSS minima when varying one by one the model parameters, in particular $x_{\mathrm{OD}}$ and $y_{\mathrm{OD}} . N$ was gradually decreased, by sub-sampling the set of data points, until the estimated OD position moved outside the visually detected OD. A number of 300 data points proved to be adequate for a correct OD identification in all the subset images and then also in the whole data set. In the four cases where OD was not correctly identified, increments of $N$ did not provide any improvement, suggesting that the problem was not linked to the number of data points used for parameter estimation.

The weights used in cost function (7) are meant to represent the (lack of) uncertainty attached to the available sample of measured direction at the specific position. Assigning a 

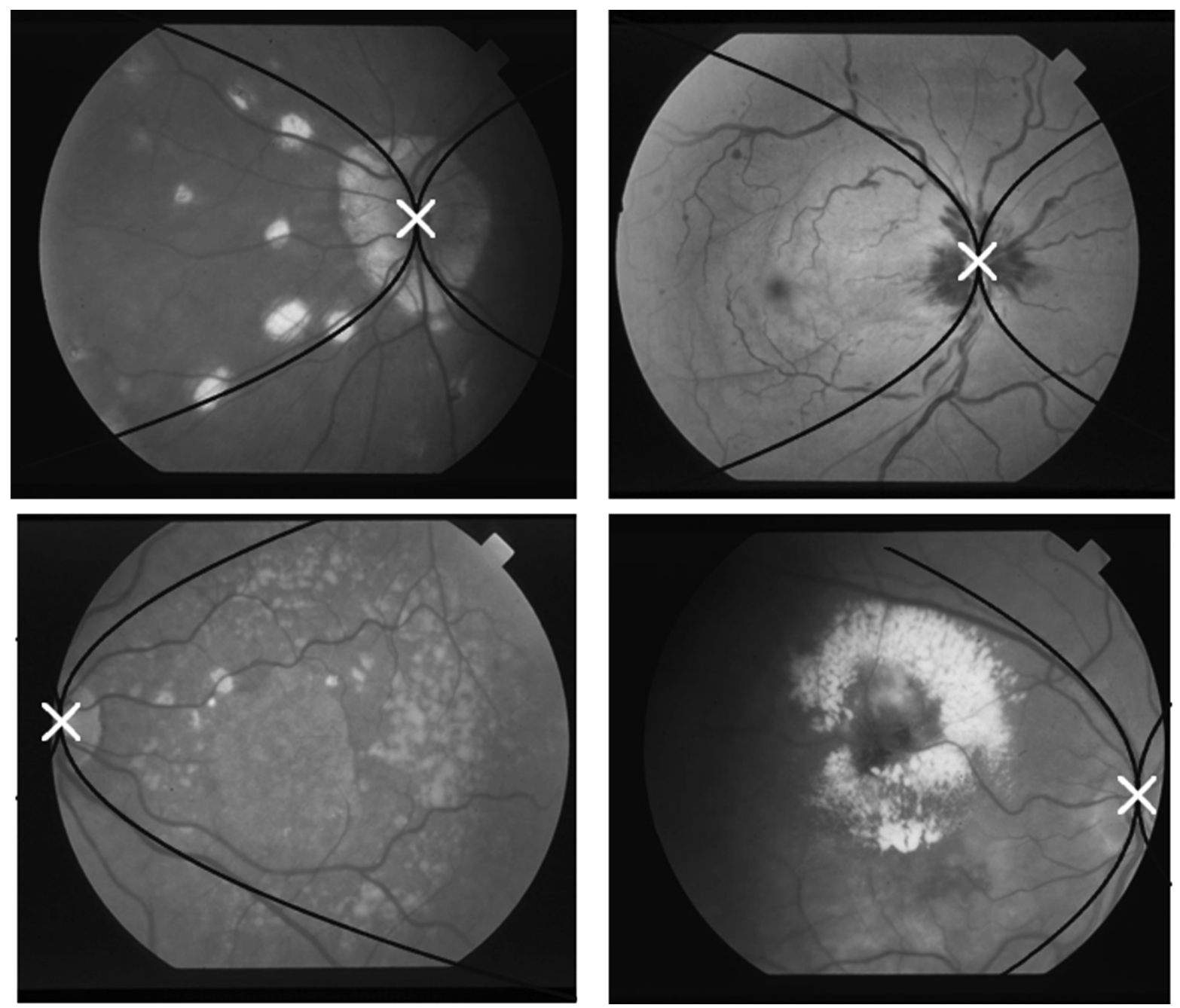

Fig. 5. Images with estimated parabolas (black line) and OD position (white cross).

higher weight to larger caliber vessels implicitly strengthens the anatomical assumption that the main vessels tend to be positioned around the parabolic locus. Smaller vessels, on the contrary, although presenting a common directional pattern between images, tend to be less regular; they are also less easily and accurately identified by vessel identification procedures and, therefore, should be assigned a higher uncertainty. If these weights were given the meaning of inverse error variances and the measured directions were normally and independently distributed around their true values, the resulting estimator would be a Maximum Likelihood estimator for the model parameters.

Results on the 81 normal and pathological images of the STARE project were satisfying, despite the presence of heavily confounding features in many of them. For example, in the images shown in Fig. 5, top, the OD is completely covered by extensive retinal lesions, either dark hemorrhages or bright exudates, and would have proved impossible to be detected by techniques based on brightness or shape. In the images of Fig. 5, bottom, the disc is only partially visible, many confounding lesions are present and only part of the vascular network is framed in the image. Thanks to its powerful model-based extrapolation capabilities, the proposed technique was able to correctly identify the position of OD also in these very difficult images. We also tested it in some images where the OD was not

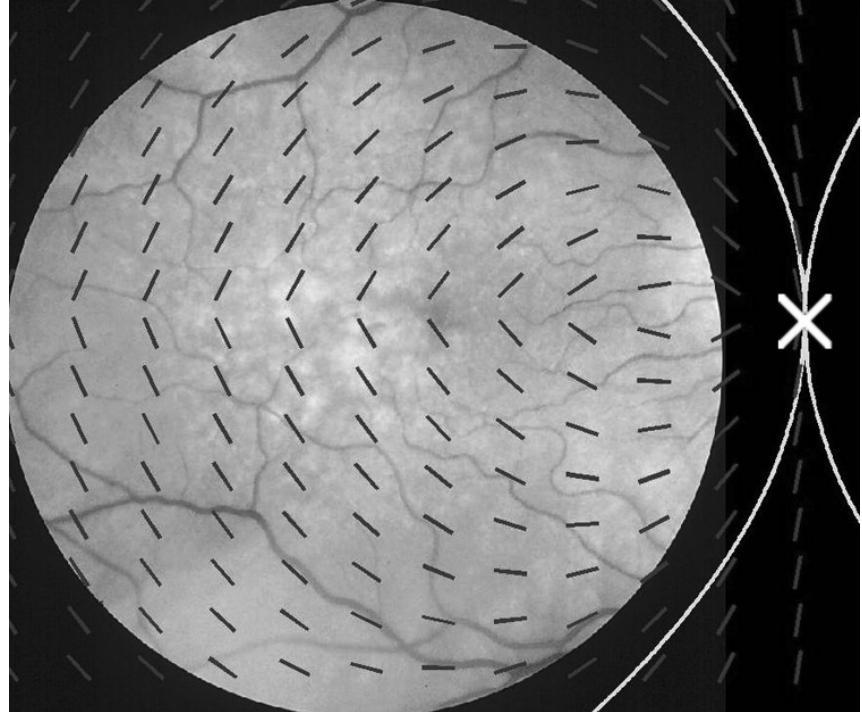

Fig. 6. Image with estimated parabolas (white line), vessel directions (dark segments) and OD position (white cross). Image not belonging to the STARE data set.

even present in the analyzed image. An example is shown in Fig. 6 (image not belonging to the STARE data base), where the 

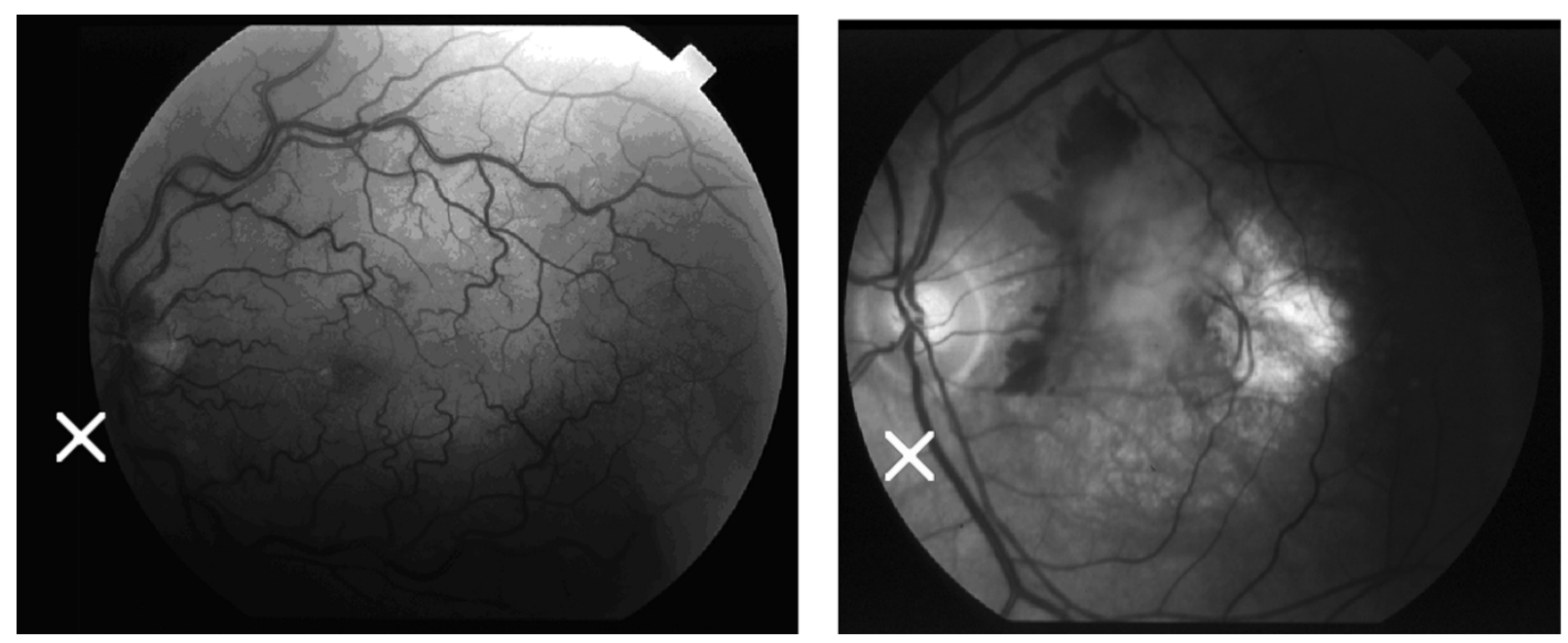

Fig. 7. Images Im0027 (left) and Im0008 (right), where estimation of OD position (white cross) using data from 'Track-1' failed.
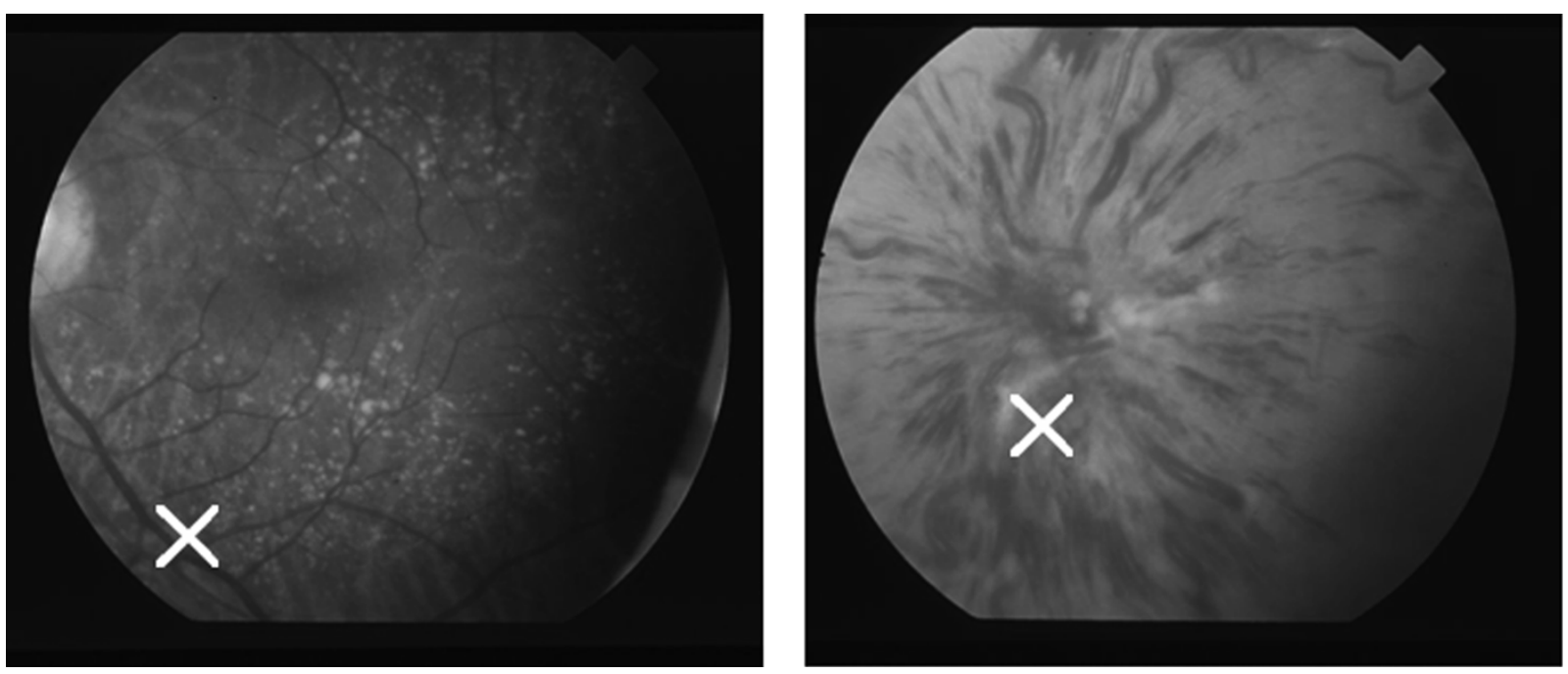

Fig. 8. Images Im0041 (left) and Im0026 (right), where estimation of OD position (white cross) using data from 'Track-2' failed.

estimated OD position is well in agreement with the location intuitively reconstructed from anatomical knowledge.

The two cases in which the OD was not correctly detected are different for "Track-1" (shown in Fig. 7) and for "Track-2" (shown in Fig. 8). The main reason for failing was the absence in the images of a sizable part of the vessel structure, e.g., for a serious pathological situation (Im0026, Fig. 8, right panel), and especially when the symmetry around the horizontal axis was totally lost [Im0027, Fig. 7 (left); Im0041, Fig. 8 (left)]. In one instance [Im0008, Fig. 7 (right)], the vascular structure was not completely reconstructed and the model predicted a slightly misplaced OD position.

On the contrary, in Im0026 with 'Track-1' data the model was able to correctly detect the OD position thanks to the erroneous recognition of radial hemorrhages as vessels. In Im0041, the different ways of recovering vessel calibers (used as weights by the parameter identification procedure) by 'Track-1' with respect to 'Track-2', allowed the former to provide from the upper part of the images (containing smaller vessels) enough data for a correct OD identification. Conversely, in both Im0027 and Im0008, data from 'Track-2' allowed a more accurate reconstruction of the vascular structure and, thus, a correct identification of model parameters.

\section{CONClusion}

The performances of the proposed method, based on a model of the vascular structure, are dependent on the availability of a good portion of this structure in the image, whereas are independent of the actual visibility (or even presence) of the OD. Being the vascular structure spread all over the image, it is much less affected by the presence of confounding or obscuring pathological areas. The availability of a vessel extraction procedure is a necessary prerequisite for our technique, and the performances of this step directly affect the correct positioning of the OD. However, the remarkably good results we obtained using the data provided by either procedure used in this work, which were algorithmically different and independently developed, suggest that this is not a critical issue.

\section{ACKNOWLEDGMENT}

The authors wish to thank Prof. A. Hoover, Clemson University, Clemson, SC, for having kindly provided the vessel identification data for the STARE images, and the STARE project for the availability on Internet of their image data set. 
Preliminary reports on this project were presented at the 3rd International Workshop on Computer Assisted Fundus Image Analysis (CAFIA3), Turin (Italy), March 2003, and at the 25th Annual International Conference of IEEE-EMBS, Cancun (Mexico), September 2003.

\section{REFERENCES}

[1] L. D. Hubbard, R. J. Brothers, W. N. King, L. X. Clegg, R. Klein, L. S. Cooper, A. R. Sharrett, M. D. Davis, and J. Cai, "Methods for evaluation of retinal microvascular abnormalities associated with hypertension/sclerosis in the atherosclerosis risk in communities study," Ophthalmology, vol. 106, pp. 2269-2280, Dec. 1999.

[2] C. Sinthanayothin, J. F. Boyce, H. L. Cook, and T. H. Williamson, "Automated localization of the optic disc, fovea, and retinal blood vessels from digital color fundus images," Br. J. Ophthalmol., vol. 83, pp. 902-910, Aug. 1999.

[3] S. F. Barrett, E. Naess, and T. Molvik, "Employing the Hough transform to locate the optic disk," Biomed. Sci. Instrum., vol. 37, pp. 81-86, Feb. 2001.

[4] $\mathrm{H}$. Li and O. Chutatape, "Automatic location of optic disk in retinal images," in Proc. IEEE-ICIP, vol. 2, Oct. 2001, pp. 837-840.

[5] M. Lalonde, M. Beaulieu, and L. Gagnon, "Fast and robust optic disk detection using pyramidal decomposition and Hausdorff-based template matching," IEEE Trans. Med. Imag., vol. 20, pp. 1193-1200, Nov. 2001.

[6] A. Osareh, M. Mirmehdi, B. Thomas, and R. Markham, "Comparison of color spaces for optic disc localization in retinal images," in Proc. 16th IEEE Int. Conf. Pattern Recognition, vol. 1, Aug. 2002, pp. 743-746.

[7] T. Walter, J. C. Klein, P. Massin, and A. Erginay, "A contribution of image processing to the diagnosis of diabetic retinopathy-Detection of exudates in color fundus images of the human retina," IEEE Trans. Med. Imag., vol. 21, pp. 1236-1243, Oct. 2002.
[8] D. Koozekanani, K. Boyer, C. Roberts, and S. Katz, "Tracking the optic nerve in OCT video using dual eigenspaces and an adaptive vascular distribution model," in Proceedings IEEE Conf. Computer Vision and Pattern Recognition, vol. 1, Dec. 2001, pp. I934-I941.

[9] A. Hoover and M. Goldbaum, "Locating the optic nerve in a retinal image using the fuzzy convergence of the blood vessels," IEEE Trans. Med. Imag., vol. 22, pp. 951-958, Aug. 2003.

[10] STARE project website. Clemson Univ., Clemson, SC. [Online]. Available: http://www.ces.clemson.edu/ ahoover/stare

[11] Y. Tolias and S. Panas, "A fuzzy vessel tracking algorithm for retinal images based on fuzzy clustering," IEEE Trans. Med. Imag., vol. 17, pp. 263-273, Apr. 1998.

[12] A. Hoover, V. Kouznetsova, and M. Goldbaum, "Locating blood vessels in retinal images by piece-wise threshold probing of a matched filter response," IEEE Trans. Med. Imag., vol. 19, pp. 203-210, Mar. 2000.

[13] M. Foracchia, E. Grisan, and A. Ruggeri, "Detection of vessel caliber irregularities in color retinal fundus images by means of fine tracking," in EMBEC'02-IFMBE Proc. Series, vol. 3, Wien, Dec. 2002, pp. $1558-1559$.

[14] N. Metropolis, A. Rosenbluth, M. Rosenbluth, A. Teller, and E. Teller, "Equation of state calculation by fast computing machines," J. Chem. Phys., vol. 21, pp. 1087-1092, 1953.

[15] ETDRS Research Group, "Grading diabetic retinopathy from stereoscopic color fundus photographs-An extension of the modified Airlie House classification,” Ophthalmology, vol. 98, pp. 786-806, May 1991. suppl..

[16] S. E. Bursell, J. D. Cavallerano, A. A. Cavallerano, A. C. Clermont, D. Birkmire-Peters, L. P. Aiello, L. M. Aiello, and The Joslin Vision Network Research Team, "Stereo nonmydriatic digital-video color retinal imaging compared with Early Treatment Diabetic Retinopathy Study seven standard field $35-\mathrm{mm}$ stereo color photos for determining level of diabetic retinopathy," Ophthalmology, vol. 108, pp. 572-585, Mar. 2001. 